\title{
High Speed, High Resolution Imaging Spectrometers Based on pnCCDs for XRF and XRD Applications
}

L. Strüder ${ }^{1,6}$, R. Hartmann ${ }^{1}$, P. Holl ${ }^{1}$, S. Ihle ${ }^{2}$, M. Huth ${ }^{2}$, J. Schmidt ${ }^{2}$, Ch. Thamm ${ }^{2}$, B. Kanngießer ${ }^{3}$, J. Baumann 3 , A. Renno ${ }^{4}$, J. Grenzer ${ }^{4}$, M. Radtke ${ }^{5}$, A. Abboud ${ }^{6}$, U. Pietsch ${ }^{6}$, H. Soltau ${ }^{2}$

1. PNSensor GmbH, Otto-Hahn-Ring 6, D-81739 Munich, Germany

2. PNDetector GmbH, Otto-Hahn-Ring 6, D-81739 Munich, Germany

3. TU Berlin, Analytical X-Ray Physics, Hardenbergstr. 36, D-10587 Berlin, Germany

4. HZDR, Bautzner Landstraße 400, 01328 Dresden, Germany

5. Bundesanstalt für Materialprüfung, Richard-Willstaetter-Str. 11, 12489 Berlin, Germany

6. University of Siegen, Walter-Flex-Str.3, 57072 Siegen, Germany

For many years pnCCDs have been well known as X-ray detectors for spectroscopic imaging in many fields of science: X-Ray Fluorescence analysis (XRF), X-ray Diffraction (XRD) with light sources in large accelerator facilities as well as with laboratory light sources or with X-rays from celestial sources in X-ray astronomy. A brief introduction in GEXRF (Grazing Emission XRF) measurements with a laboratory laser produced plasma source will be given, PIXE (Particle Induced X-ray Emission) measurements and D2XRF (Double Dispersive X-Ray Fluorescence) and Slicing experiments with pnCCDs coupled to polycapillary optics performed at the BESSY synchrotron will be shown. Energy-dispersive Laue diffraction with ultra-hard X-rays for the analysis of defects in metals will conclude the overview of spectroscopic X-ray imaging measurements in the field of structure and dynamics of matter.

pnCCDs are radiation detectors on high resistivity $450 \mu \mathrm{m}$ thick fully sensitive silicon [1]. They are back-illuminated devices with an ultra-thin, homogeneous radiation entrance window, enabling the proper detection of X-rays up to $30 \mathrm{keV}$ with high quantum efficiency. As all pnCCDs are equipped with a fully column parallel readout, frame rates on more than 1200 frames per second are achieved, keeping the read noise level at 3 electrons (rms). Some of the key performance figures are (1) a quantum efficiency above $90 \%$ from $1 \mathrm{keV}$ up to $10 \mathrm{keV}$, (2) single photon counting capability starting at only $30 \mathrm{eV}$, (3) extreme radiation hardness due to the avoidance of active MOS structures, and (4) energy resolution of $130 \mathrm{eV}$ (FWHM) at $6 \mathrm{keV}$ and $37 \mathrm{eV}$ (FWHM) at $90 \mathrm{eV}$. These properties have enabled a variety of spectacular measurements.

(a) GEXRF: By combining a highly brilliant laser produced plasma (LPP) source with a scanningfree setup, grazing emission X-ray fluorescence (GEXRF) measurements in the soft X-ray range were realized [2]. The detector, a pnCCD, was operated in a single photon counting mode in order to utilize its energy dispersive properties. GEXRF profiles of the Ni-L $\mathrm{L}_{\alpha}$ line of a carbon-nickel multilayer sample, which displays a lateral (bi-)layer thickness gradient, were recorded at several positions. Simulations of theoretical profiles predicted a prominent intensity dip at emission angles between $5^{\circ}$ and $12^{\circ}$, depending strongly on the bi-layer thickness of the sample (see Fig.1). This information was used to retrieve the bi-layer thickness gradient. The results are in good agreement with values obtained by X-ray reflectometry, conventional X-ray fluorescence and transmission electron microscopy measurements and serve as proof of principle for the suggested GEXRF setup.

(b) PIXE: The unique properties pnCCDs, coupled to polycapillary X-ray optics, allows a fast position resolved overview over a large detection area with first results visible in real time. The maximum field of view exceeds $1 \mathrm{~cm}^{2}$ and the spatial resolution approaches a few microns when 
using sub-pixel algorithms by centroiding the signal charge cloud in the pixel structure [3]. The device has been used as an X-ray detector at the PIXE beamline at the Helmholtz-Zentrum Dresden Rossendorf (see Fig. 2).

In addition to the above measurements (c) D2XRF and Slicing experiments performed at the BESSY synchrotron will be presented (see Fig. 3) as well as (d) Energy Dispersive Hard X-ray Laue Diffraction measurements at the ESRF.

References:

[1] I. Ordavo et al., NIM A 654, (2011), 250 - 257

[2] J. Baumann et al., submitted to Applied Physics Letters (2016)

[3] S. Novak et al., X-ray Spectrometry, 44(3), 2015

[4] M. Radtke et al., Microchemical Journal 125 (2016) 56-61

[5] A. Abboud et al., Rev. of Sci. Instr., Volume 85, Issue 11 (2014)

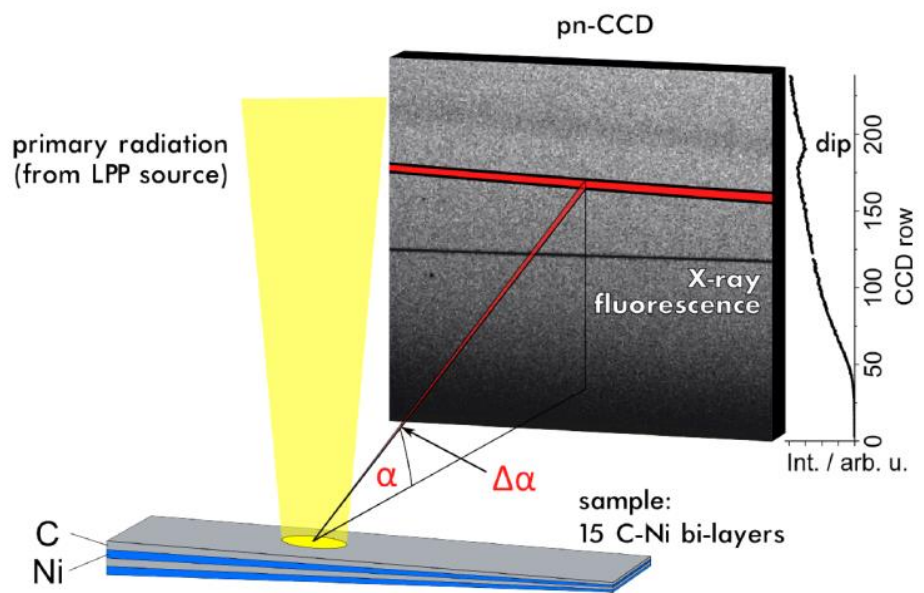

Fig.1: Scheme of the scanning free GEXRF setup. The monochromatized radiation of a LPP source is focused onto the sample plane with an incident angle of about $90^{\circ}$. The fluorescence radiation created in the $\mathrm{C}-\mathrm{Ni}$ multilayer sample is recorded at shallow angles with a pnCCD acting as energy dispersive area detector. An interference pattern is visible in the image as horizontal intensity dip [2].
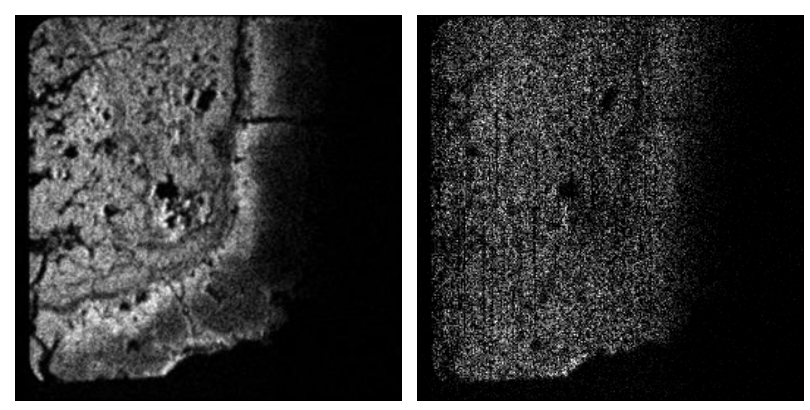

Fig. 2: Result from a PIXE measurement with trace element distributions of $\mathrm{Cu}$ (left) and $\mathrm{Sn}$ (right) in Mn nodules. The proton energy was 3 $\mathrm{MeV}$, the beam current $1 \mu \mathrm{A}$. „Extracted“ qualitative trace element distribution in one frame $\left(12 \times 12 \mathrm{~mm}^{2}\right)$ of a typical high speedPIXE measurement is shown. The 2D distribution of the fluorescence signals is displayed in real time throughout the measurement.

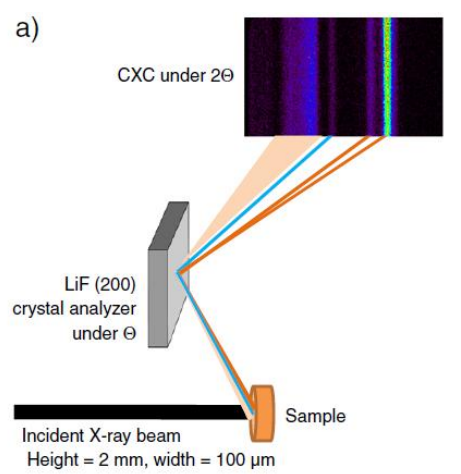

b)

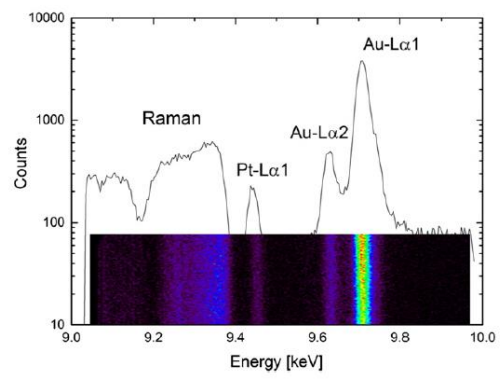

Fig. 3: Sketch for D2XRF measurements for the detection of Pt in Au: (a) with the contribution of all lines: trace element (blue), major element (orange) and the respective XRRS (light orange). (b) Measurement of different lines from $\mathrm{Pt}$ and Au. The XRRS contribution can be properly distinguished [3]. 